RESEARCH ETHICS

\title{
Parental preferences for neonatal resuscitation research consent: a pilot study
}

A Culbert, D J Davis

J Med Ethics 2005;31:721-726. doi: 10.1136/jme.2004.011247

See end of article for authors' affiliations

.....................

Correspondence to: Dr D J Davis, Center for Clinical Education, Teilum, Afsnit 5404 ,

Rigshospitalet

Blegdamsvej 9,2100

Copenhagen O, Denmark; deborah.davis@rh.hosp.

dk

Received

17 November 2004

In revised form

31 March 2005

Accepted for publication

4 April 2005 Objective: Obtaining informed consent for resuscitation research, especially in the newborn, is
problematic. This study aimed to evaluate parental preferences for hypothetical consent procedures in neonatal resuscitation research.

Design: Mail-out survey questionnaire.

Setting/participants: Randomly selected parents who had received obstetrical or neonatal care at a tertiary perinatal centre.

Main outcome measures: Parental levels of comfort (Likert-type scale 1-6) regarding different methods of obtaining consent in hypothetical resuscitation research scenarios.

Results: The response rate was $34 \%$. The respondents were a group of highly educated women with a higher family income than would be expected in the general population. In terms of results, parents valued the impact the research would have on their baby and the importance of a positive interaction with the physicians conducting the research study. Parents felt most comfortable with prospective consent in the setting of prenatal classes or prenatal visits with a physician, but they were somewhat uncomfortable with prospective consent upon admission to hospital after labour had begun. Parents were uncomfortable with waived consent, deferred consent, and opting out, no matter when during the pregnancy consent was requested.

Conclusion: This pilot study reports parental preferences for prenatal information and consent for such research trials of neonatal resuscitation. A low response rate and potentially skewed demographics of the respondents prevent generalisability of this result. Interview studies should be performed to better determine parental preferences for informed consent in a more representative population.
$\mathrm{F}$ ew of the resuscitation procedures, drugs, or treatments currently in use or speculated to be of use in the future, have been put to the test of randomised controlled trials in neonates. Paramount to the conduct of randomised controlled trials is valid informed consent, but this may be problematic for neonatal resuscitation research. As new resuscitation methods are embarked upon, it would be important to ensure appropriate informed consent. At the time of delivery the parent(s) who act as the surrogate decision maker may not be in a medical or psychological condition to be able to understand the information given to them and to make an informed choice for their child in a timely fashion. Although a few studies ${ }^{1-3}$ have evaluated informed consent given by parents for their newborns to participate in a variety of different research protocols, consent procedures in neonatal resuscitation research or in urgent situations have not specifically been studied. We therefore designed a survey questionnaire for parents to determine their preferences for consent procedures in neonatal resuscitation research. A key feature of this pilot study was a series of hypothetical scenarios using different models of obtaining consent to which parents gave their preferences.

\section{METHODS}

\section{Study population}

Invitations to participate in the study were sent to 318 parents who had received care in a regional perinatal centre in Canada. Invitees included parents of babies previously admitted to the neonatal intensive care unit (NICU), parents of babies previously admitted to the normal newborn nursery, and parents-to-be in the second trimester of pregnancy. The first two groups of invitees were selected randomly from all parents whose babies had been admitted to the hospital, and for the latter group the parents-to-be were selected sequentially when they presented to the clinic in the second trimester of pregnancy.

\section{Questionnaire development}

The questionnaire was developed from comments of focus groups of NICU parents of small preterm infants, nursing staff at an inborn NICU at a regional perinatal centre, and nursing staff at an outborn NICU at a children's hospital. Drafts of the questionnaire were reviewed by 10 former NICU parents and five neonatologists and appropriate revisions were made. Demographic information and opinions on what parents felt would be important in helping them decide whether to enrol their child in a research project were surveyed. Parents were asked to indicate their comfort with the consent process in a series of 15 hypothetical situations depicting various methods of obtaining consent, including rushed with limited information, prospective, waived, deferred, and opting out forms of consent (Appendix 1), on a Likert-type scale from very comfortable to very uncomfortable.

\section{Mail-out protocol}

A letter was sent to eligible individuals inviting participation; return of the signed consent form indicated willingness to participate. Those who returned the consent form were mailed a survey in French or English. Approximately one week after the initial mailing, a reminder letter was sent. If the survey had not been returned within four weeks, a replacement was sent. If a completed survey was still not returned after this mailing, no further contact was made. 


\section{Ethical approval}

The study was approved by the research ethics boards at the perinatal centre and the children's hospital.

\section{Data analysis}

For the analysis, comfort levels were assigned numerical scores (very comfortable $=1$, comfortable $=2$, somewhat comfortable $=3$, somewhat uncomfortable $=4$, uncomfortable $=5$, very uncomfortable $=6$ ). Comfort levels for consent procedures in the hypothetical scenarios were compared using the Wilcoxon rank sum test with significance defined as $\mathrm{z}$ value $<0.05$ at $0.000 \times$.

\section{RESULTS}

\section{Response rate}

Of 107 individuals who agreed to participate, 102 (95\%) completed the survey (81 mothers, 21 fathers). The response rate to the invitation to participate was $34 \%$ ( 107 of 318 parents) with an equivalent distribution between NICU parents $(n=37)$, normal newborn nursery parents $(n=39)$ and parents-to-be $(\mathrm{n}=31)$. No differences in comfort levels to the different hypothetical scenarios were detected between the three subgroups, so the combined results of respondents are reported.

\section{Demographic data}

The average age of the respondents was 32.5 years and more than three quarters were married. Respondents had an average of 1.5 children. Seventy seven per cent had a community college degree or higher and the median family income was between CAN\$50 000 to CAN\$74 999 .

\section{Experiences with acute care and medical research}

Nineteen parents had experienced the death of a child. More NICU parents $(4 / 36,11 \%)$ reported having a child with a disability than did normal newborn nursery parents $(1 / 37$, $2.7 \%)$ and parents-to-be $(2 / 29,7 \%)$. Fourteen parents had agreed in the past to participate in medical research and 14 had agreed for a loved one to participate in medical research.

\section{Factors identified as important in decision-making}

The factors that were most often rated as important or very important in deciding whether or not to consent to their child's participation in research included: knowing that being in the study would not cause your baby more pain or suffering; feeling that the doctors would act in the best interest of your baby; feeling that you understood the study; feeling that you were able to ask questions and receive honest answers; trusting the individuals explaining the study to you; having written material about the study available; feeling that the study drug was likely to help your baby; and having sufficient time to think about whether or not you wished your baby to participate in the study. Previous research done with the study drug or procedure and approval by various types of ethics committees did not rank highly in importance.

\section{Reactions to the hypothetical consent scenarios}

The five hypothetical consent scenarios with which parents felt most comfortable are shown in table 1.

Parents preferred the scenarios in which consent was obtained prospectively. Greater than $50 \%$ of respondents were comfortable or very comfortable with granting consent at prenatal visits $(55 / 102)$ or in prenatal classes (53/102). Consent during a prenatal visit was especially preferred for complicated pregnancies, with two thirds of respondents (69/ 102) choosing comfortable or very comfortable. For all scenarios of prospective consent, respondents appreciated the full explanation in advance and the time to think about whether they wanted their baby to participate. Limitations to this approach mentioned by parents included: discomfort in consenting to something that had not yet happened; inability to make a proper decision until the situation applied directly; and the possibility of provoking unnecessary anxiety. Concerns were also raised about whether physicians had adequate time to give information about research studies at prenatal visits. For example, one parent commented, "Physicians often do not have time to explain and answer questions." Some also indicated they would prefer that information be given by physicians directly involved in the study. Prenatal classes were felt to have some limitations as venues for information or consent because not all women attend.

Despite the preference for prospective consent, parents overall felt somewhat comfortable (median response 3) with consent at the time of resuscitation without prior information. A third of respondents (32/102) indicated that they were comfortable or very comfortable with this scenario. Although parents commented they would have preferred to have the study explained in advance and to have additional time to think about it, they seemed to appreciate that in an urgent situation this type of consent might be the only option. Nonetheless, they indicated that they would not like being approached at a time of duress and how it would be difficult to concentrate and make a properly informed decision. Consent at the time of resuscitation with prior explanation at a prenatal visit increased the comfort level significantly from 3 to 2 ( $z$ value of less than 0.05 at $0.000 \times$ ); just over half of all respondents (53/102) indicating that they were comfortable or very comfortable with this scenario. Comments indicated appreciation with having prior knowledge of the study. Some respondents would have preferred to give consent in advance, rather than just receive information.

The scenarios with which parents felt somewhat uncomfortable or worse are shown in table 2. Once labour had begun parents stressed that it would be difficult to absorb important information and make an important decision about study participation. Respondents indicated that they would immediately start to worry that something was wrong and that information about their baby's condition was being withheld.

Table 1 Hypothetical resuscitation scenarios with which the parents felt at least somewhat comfortable, presented in descending order

\begin{tabular}{ll}
\hline Brief description & $\begin{array}{l}\text { Median } \\
\text { comfort }\end{array}$ \\
\hline Information and consent at prenatal visit. Complicated pregnancy (scenario 13) & 2 \\
Information and consent at prenatal visit. Uncomplicated pregnancy (scenario 6) & 2 \\
Information at prenatal visit. Consent when baby requires resuscitation (scenario 15) & 2 \\
Information and consent at prenatal class (scenario 5) & 2 \\
Consent when baby requires resuscitation. No prior information (scenario 2) & 3
\end{tabular}

Comfort levels were indicated on a Likert-type scale: $1=$ very comfortable, $2=$ comfortable, $3=$ somewhat comfortable, $4=$ somewhat uncomfortable, $5=$ uncomfortable, $6=$ very uncomfortable. 
Table 2 Hypothetical scenarios with which parents felt somewhat uncomfortable or worse, presented in descending order of comfort level

\begin{tabular}{ll}
\hline Brief description & $\begin{array}{l}\text { Median } \\
\text { comfort }\end{array}$ \\
\hline Information and consent during labour. Complicated pregnancy (scenario 11) & 4 \\
Information and consent during labour. Uncomplicated pregnancy (scenario 8) & 4 \\
Deferred consent, baby is alive (scenario 3) & 4 \\
Waived consent (scenario 1) & 4 \\
Opting out at prenatal visit. Complicated pregnancy (scenario 14) & 4 \\
Opting out at prenatal visit. Uncomplicated pregnancy (scenario 7) & 4 \\
Deferred consent, baby has died (scenario 4) & 5 \\
Opting out during labour. Complicated pregnancy (scenario 12) & 5 \\
Opting out during labour. Uncomplicated pregnancy (scenario 9) & 5 \\
Opting out presented in media format (scenario 10) & 5 \\
\hline
\end{tabular}

Comfort levels were indicated on a Likert-type scale: 1 =very comfortable, 2 =comfortable, $3=$ somewhat comfortable, $4=$ somewhat uncomfortable, $5=$ uncomfortable, $6=$ very uncomfortable.

For a scenario of waived consent, there was a bimodal distribution in comfort levels. The majority felt uncomfortable and indicated a desire to be informed and to give consent for any experimental procedures on their child. A smaller number of respondents felt comfortable with waived consent and indicated that they assumed physicians would act in the best interests of their child.

Forty four of the 102 respondents were uncomfortable or very uncomfortable with deferred consent, even if the baby were still alive. They were unhappy not having a choice in whether their baby participated. They commented that they should have been provided with information about the study prior to the crisis. Those who were comfortable felt that doctors would act in the best interests of their baby and were reassured that the drug seemed to have shown positive results given the baby's survival to that point in time. The comfort level dropped significantly ( $\mathrm{z}$ value of less than 0.05 at $0.000 \times$ ) for deferred consent if the baby had died. Parents interpreted the death of the baby as an indication that the drug potentially contributed to the outcome, and more than half indicated they would feel very uncomfortable with this scenario.

Respondents were most uncomfortable with opting out as a means of providing consent. Respondents did not like automatic inclusion of their baby in a research study and felt that consent should be required not assumed. Respondents expressed that they wanted to make decisions and provide consent in order for their baby to be included in any research study.

\section{DISCUSSION}

As many as 25/1000 live born term infants will have some degree of fetal asphyxia (defined as a base deficit $\geqslant 12 \mathrm{mmol} /$ 1). Up to $4 / 1000$ have moderate to severe fetal asphyxia with the potential for significant sequelae (base deficit of $\geqslant 12 \mathrm{mmol} / \mathrm{l}$ plus encephalopathy with or without multiorgan dysfunction). ${ }^{4}$ With advances in our understanding of the pathogenesis of brain damage, new treatments for fetal asphyxia are sure to emerge. For therapy to be of benefit it will have to be administered during or very soon after the insult. With this emphasis placed on early intervention, time to discuss a resuscitation research study with the parents of a newborn is lacking, which will make ethically valid informed consent problematic. Issues include how to protect the rights of the child and fully inform parents as they decide about their child's participation but not overburden them at a time of great psychological distress. ${ }^{5}$ As has been shown by others, ${ }^{13}$ parents want to decide about participation of their babies in research. The parents in our pilot study were similarly quite adamant about their role in decision making for their offspring.
Parents in our study clearly expressed most comfort in consent to a neonatal resuscitation research study if they had received information about the study in advance, that is during a prenatal visit or prenatal class, and had time to understand and discuss it with their loved ones and/or physician. Certainly, such prospective consent allows for the three important elements of disclosure, capacity, and voluntariness, to be fulfilled. ${ }^{6}$ However, there are issues of retention of information if the background about a research study is given to potential participants well ahead of time. Unpublished data from another pilot study done by our group indicates that preferences for consent to hypothetical situations does not change between the second trimester of gestation and six weeks postpartum. However, little is known about the length of retention, and the quantity and the quality of retained information about a research study if such information is given in advance. Such studies would be necessary.

A further ethical question would be whether prospective consent would be valid if it were obtained at a time when the potential subject of the future research were a fetus, and therefore not a legal entity in most jurisdictions. Given that parents were comfortable both with prospective consent and with prospective information followed by consent at the time the intervention was needed, making the study information available prenatally but obtaining the consent only if the clinical situation warranted may be the more appropriate option.

In their comments, a few parents expressed concern that prospective consent could unnecessarily worry some parentsto-be. Ethically, researchers would have to balance the potential harm of this undue worry for some parents with the potential benefit of the improved information to the few whose ability to provide informed consent would be improved by the prior information.

Parents were uncomfortable with deferred consent, waived consent, and opting out, which have been touted as potential forms of consent for resuscitation research. This discomfort reflected their wish for choice in what happens to their offspring. The parents said they would be uncomfortable if they did not have rights of refusal or withdrawal. In contrast, other studies have found acceptance of deferred consent to be good among family members of adult patients enrolled in resuscitation research. ${ }^{7}$ Deferred consent seemed to have good acceptance in a study of oxygen versus room air for neonatal resuscitation. ${ }^{8}$ Opponents of this consent method have argued that the decision to continue in the research study cannot be used to support the claim that there was or would have been consent to the initiation of the research.' Deferred consent does not support the element of voluntariness. ${ }^{6}$ 
Waived consent has also been put forth as a method of obtaining consent for resuscitation research. An independent, informed group of experts in the field could assess a research proposal and deem it safe thereby eliminating the need for informed consent to be obtained from research subjects. Defendants argue that this eliminates the emotional and stressful burden placed on family members at the time of an acute and life threatening situation. ${ }^{10} 11$ The US Food and Drug Administration requirements for waiver of consent include that the situation is life threatening, the patient is unable to communicate, and there is insufficient time to obtain informed consent. ${ }^{12}$ Neonatal resuscitation can certainly fit this definition. However, parents in our study were uncomfortable with waived consent and clearly wanted to be informed and make the decision for their child to participate.

We did not include a scenario with a Zelen pre-randomised consent, in which consent is requested after randomisation. ${ }^{13}$ We do not know if parents in our study would have felt comfortable with this approach. Statistical and ethical issues of concern with pre-randomisation consent have been noted previously. ${ }^{14}$ Furthermore, this approach would not overcome the problems associated with informed consent in resuscitation noted above, that is issues around disclosure, capacity, and voluntariness.

Opting out has been suggested as an alternative to informed consent and has proved to be acceptable in studies that do not increase patient risk. ${ }^{15}{ }^{16}$ Manning has suggested that informing parents in the antenatal period about the possible need for emergency neonatal research with presumed consent and the possibility to opt out would be ethically sound, might increase enrolment, and might decrease selection bias. ${ }^{17}$ However, parents in our study were very uncomfortable with the opting out scenarios and wanted to provide consent, not have it presumed for them.

\section{Limitations}

Our study provides interesting information about parental preferences for consent in neonatal resuscitation research, however, there are some obvious severe limitations. Although 95\% who agreed to participate completed the survey, the response to the invitation to participate was low at only $34 \%$. Furthermore, the respondents were not representative of the general population: most were married, highly educated women with a high family income. A high number of respondents had also lost a baby and may have felt emotionally connected to the subject material and wanted to share their experiences. Those who had had previous experiences with neonatal resuscitation may have been more inclined to participate. Because of these unanticipated demographics of the respondents, no generalisable conclusions can be drawn from this pilot study. A study population with a lower socioeconomic profile may give quite different responses. We suggest that an interview protocol would be necessary to better ensure a more representative population than our mail-out survey. Another obvious limitation is that the consent scenarios were hypothetical. Parental responses to real-life personal situations of consent to neonatal resuscitation research may well be different.

\section{CONCLUSION}

Although our study suggests that parents appear most comfortable with prospective consent, one cannot underestimate the logistical challenge of such an undertaking. Furthermore, prospective consent would only be possible where pregnant women have access to prenatal care and visit regularly with a physician throughout their pregnancy. Since over half of the parents were in fact at least somewhat comfortable with the current method of obtaining consent for urgent neonatal research, in a rush without prior information, one might ask whether anything further needs to be done. We would argue that if individual autonomy is something that we value and respect then surely we must search for better ways of obtaining consent in emergency situations. Without this respect we are unlikely to foster the critical partnership between researcher and research subject, which is vital as we work together to further medical knowledge and develop improved treatment options. This pilot study lays some of the groundwork for further research in parental preferences to consent for urgent neonatal research. However, an interview protocol should be trialled to ensure a larger sample size with more representative demographics prior to being able to definitively comment on parental preferences for such difficult tasks as true informed consent for neonatal resuscitation research.

\section{ACKNOWLEDGEMENTS}

We wish to thank all the parents who completed the surveys. For many, it was clear that their pregnancy and birthing experiences were highly emotional, often accompanied by loss. Their willingness to share their thoughts and comments is truly appreciated.

\section{Authors' affiliations \\ A Culbert, D J Davis, Departments of Pediatrics and Obstetrics/ Gynecology, University of Ottawa, Ottawa, Ontario, Canada}

This research was supported by a grant from the American Academy of Pediatrics NRP Research Grant Program

\section{APPENDIX 1}

\section{HYPOTHETICAL SCENARIOS INCLUDED IN THE SURVEY QUESTIONNAIRE}

Parents responded to the question "How comfortable would you feel with this situation?" by marking one of six boxes (very comfortable, comfortable, somewhat comfortable, somewhat uncomfortable, uncomfortable, very uncomfortable). For the analysis, these were assigned values of 1 (very comfortable) to 6 (very uncomfortable). There was also an open-ended question, "Why?" following each set of response boxes.

(1) After an uncomplicated pregnancy, you or your partner delivers a baby who unexpectedly at the time of birth is not breathing and has no heart rate. The health care team tries all of the usual things to get the baby breathing and the heart pumping without good success. Because they think it might give the baby a better chance, they give the baby a commonly recommended drug, but the dose they use is very different from the usual dose. They don't tell you they are going to try the drug before doing it, but it is part of a study approved by the hospital Research Ethics Committee.

(2) After an uncomplicated pregnancy, you or your partner delivers a baby who unexpectedly at the time of birth is not breathing and has no heart rate. The health care team tries all of the usual things to get the baby breathing and the heart pumping without good success. The doctor rushes in and tells you that the hospital is taking part in a study of a drug for babies who may not have had enough oxygen at birth. The study drug must be given within 30 minutes of birth. He or she asks you to sign a consent form for the study.

(3) After an uncomplicated pregnancy, you or your partner delivers a baby who unexpectedly at the time of birth is not breathing and has no heart rate. The health care team tries all of the usual things to get the baby breathing and the heart pumping without good success. Finally the doctors decide to give the baby a study drug and the baby responds but remains in critical condition. 
Later a doctor comes to talk to you and tells you that while trying to get the baby breathing and the heart pumping, the baby had been entered in a study of a drug for babies who have had a lack of oxygen at birth. The doctor asks you to sign a consent form for the study.

(4) After an uncomplicated pregnancy, you or your partner delivers a baby who unexpectedly at the time of birth is not breathing and has no heart rate. The health care team tries all of the usual things to get the baby breathing and the heart pumping without good success. Finally the doctors decide to give the baby a study drug but the baby dies anyway. Several hours later, a doctor comes to talk to you and tells you that during the efforts to get the baby breathing and the heart pumping, the baby had been entered in a study of a drug for babies who have had a lack of oxygen at birth. The doctor asks you to sign a consent form for the study.

(5) You are an expectant parent attending a prenatal class. The instructor has invited a pediatrician who works at the hospital where you or your partner will deliver to speak to your group. The pediatrician describes a study that is ongoing at the hospital to test a drug for babies who have a lack of oxygen at birth. He or she answers questions that the group has. Finally, he or she asks all of you who are interested to sign a consent form for the study, just in case your baby is eligible to enter the study but there is not time to discuss it when the baby is having difficulty.

(6) You or your partner are visiting the doctor for a prenatal visit. The doctor tells you that there is a study ongoing at the hospital to test a drug for babies who have a lack of oxygen at birth. He or she gives you a copy of the information about the study and asks you to sign a consent form for the study, just in case your baby is eligible to enter the study but there is not time to discuss it when the baby has difficulty.

(7) You are your partner are visiting the doctor for a prenatal visit. The doctor tells you that there is a study ongoing at the hospital to test a drug for babies who have had a lack of oxygen at birth. He or she gives you a copy of the information about the study. He or she says that your baby will be entered in the study unless you "opt out" by signing a form. He or she explains that this is necessary because the drug needs to be given soon after birth and there wouldn't be time to talk about the study then. If you sign the form, your baby, even if eligible, will not be given the study drug. If you don't sign this form, your baby, if eligible, will be given the study drug.

(8) You or your partner goes to the hospital excited to finally be in labour after a pregnancy that has gone very well. The contractions are coming every 5 minutes. Following the usual admission procedure, an individual comes to talk to you about a study to test a drug for babies who have had a lack of oxygen at birth. He or she asks you to sign a consent form for the study, just in case your baby is eligible, explaining that the drug needs to be given soon after birth and there wouldn't be time to talk about the study then.

(9) You or your partner goes to the hospital excited to finally be in labour after a pregnancy that has gone very well. The contractions are coming every 5 minutes. Following the usual admission procedure, an individual comes to talk to you about a study to test a drug for babies who have had a lack of oxygen at birth. He or she tells you that all eligible babies will be entered in the study unless their parents sign a form to "opt out" of the study. He or she explains that this is necessary because the drug needs to be given soon after birth and there wouldn't be time to talk about the study then. If you sign the form, your baby, even if eligible, will not be given the study drug. If you don't sign this form, your baby, if eligible, will be given the study drug.

(10) You or your partner is due to deliver a baby at your local hospital. On the radio, on television, or in the newspaper, you hear about a study going on at the hospital. The study will test a drug for babies who have had a lack of oxygen at birth. The announcements explain that if parents do not want their baby entered, they should ask to sign a form to "opt out" of the study before the baby delivers. If they don't sign this form, their baby will automatically be entered in the study if eligible. They give a number you can call for more information.

(11) After a pregnancy that has had many complications, you or your partner enters the hospital in labour. The contractions are coming every 5 minutes. Following the usual admission procedure, an individual comes to talk to you about a study to test a drug for babies who have had a lack of oxygen at birth. He or she asks you to sign a consent form just in case your baby is eligible. He or she explains that this is necessary because the drug needs to be given soon after birth and there wouldn't be time to talk about the study then.

(12) After a pregnancy that has had many complications, you or your partner enters the hospital in labour. The contractions are coming every 5 minutes. Following the usual admission procedure, an individual comes to talk to you about a study to test a drug for babies who have had a lack of oxygen at birth. He or she tells you that all eligible babies will be entered in the study unless the parents sign a form to "opt out" of the study. He or she explains that this is necessary because the drug needs to be given soon after birth and there wouldn't be time to talk about the study then. If you sign the form, your baby, even if eligible, will not be given the study drug. If you don't sign this form, your baby, if eligible will be given the study drug.

(13) You or your partner are visiting the doctor for a prenatal visit during a pregnancy that has had many complications. The doctor tells you that there is a study ongoing at the hospital to test a drug for babies who have had a lack of oxygen at birth. He or she gives you a copy of the information about the study ands asks you to sign a consent form for the study, just in case your baby is eligible to enter the study but there is not time to discuss it when the baby has difficulty.

(14) You or your partner are visiting the doctor for a prenatal visit during a pregnancy that has had many complications. The doctor tells you that there is a study ongoing at the hospital to test a drug for babies who have had a lack of oxygen at birth. He or she gives you a copy of the information about the study. He or she says that your baby will be entered in the study unless you "opt out" by signing a form, explaining that if your baby is eligible to enter the study there will not be time to discuss it when the baby has difficulty. If you sign the form, your baby, even if eligible, will not be given the study drug. If you don't sign this form, your baby, if eligible, will be given the study drug.

(15) You or your partner visit the doctor for a prenatal visit. The doctor tells you that there is a study ongoing at the hospital where delivery is planned to test a drug for babies who have had a lack of oxygen at birth. He or she gives you a copy of the information about the study 
which gives details about the study, including the risks and benefits. There is also a number you can call for more information. The pregnancy continues and labour starts within about 2 weeks of your due date. Unexpectedly at the time of birth your baby is not breathing and has no heart rate. The health care team tries all the usual things to get the baby breathing and the heart pumping without good success. The doctor rushes in and asks you to sign a consent form for the study that your doctor told you about.

\section{REFERENCES}

1 Mason SA, Allmark PJ, for the Euricon Study Group. Obtaining informed consent to neonatal randomized controlled trials: Interviews with parents and clinicians in the Euricon study. Lancet 2000;356:2045-51.

2 Zupancic JAF, Gillie P, Streiner DL, et al. Determinants of parental authorization for involvement of newborn infants in clinical trials. Pediatrics 1997;99:e6.

3 Burgess E, Singhal N, Amin H, et al. Consent for clinical research in the neonatal intensive care unit: a retrospective survey and a prospective study. Arch Dis Child Fetal Newborn Ed 2003;88:F280-5.

4 Low JA. Determining the contribution of asphyxia to brain damage in the neonate. J Obstet Gynaecol Res 2004;30:276-86.
5 Mason S A Obtaining informed consent for neonatal randomized controlled trials - an "elaborate ritual"? Arch Dis Child 1997;76:F143-5.

6 Etchells E, Sharpe G, Walsh P, et al. Bioethics for clinicians: 1. Consent. Can Med Assoc J 1996;155:177-80.

7 Abramson NS, Safar P. Deferred consent: Use in clinical resuscitation research. Ann Emerg Med 1990;19:781-4.

8 Saugstad OD, Rootwelt T, Aalen O. Resuscitation of asphyxiated newborn infants with room air or oxygen: an international controlled trial: the Resair 2 study. Pediatrics 1998;102:e1.

9 Miller BL. The ethics of cardiac arrest research. Ann Emerg Med 1993;22:188-24

10 Biros MH, Lewis RJ, Olson CM, et al. Informed consent in emergency research. JAMA 1995;273:1283-7.

11 Spivey WH, Abramson NS, Iserson KV, et al. Informed consent for biomedical research in acute care medicine. Ann Emerg Med 1991;20:1251-65.

12 US Food and Drug Administration Dept of Health and Human Services. Protection of human subjects: informed consent. Federal Register 1995;60:49086-103

13 Zelen M. Randomized consent designs for clinical trials: An update. Stat Med 1990;9:645-56.

14 Allmark P. Should Zelen pre-randomized consent designs be used in some neonatal trials? J Med Ethics 1999;25:325-9.

15 Rogers CG, Tyson JE, Kennedy KA, et al. Conventional consent with opting in versus simplified consent with opting out: An exploratory trial for studies that do not increase patient risk. J Pediatr 1998;132:606-11.

16 Mutch L, King R. Obtaining parental consent-opting in or opting out? Arch Dis Child 1985;60:979-80.

17 Manning DJ. Presumed consent in emergency neonatal research. J Med Ethics 2000;26:249-53.

\section{1 th European Forum on Quality Improvement in Health Care}

26-28 April 2006, Prague, Czech Republic

For further information please go to: www.quality.bmipg.com

Book early to benefit from a discounted delegate rate 